\title{
TRACTOGRAFÍA Y TENSOR DE DIFUSIÓN EN EL ESTUDIO POR RESONANCIA MAGNÉTICA DEL NERVIO MEDIANO. REPORTE DE UN CASO
}

\author{
Drs. Paulina Yañez $S^{(1)}$, Yessenia Orellana $A^{(2)}$, TM. Daniela Vega $T^{(3)}$, Dr. Marco Naranjo $\boldsymbol{R}^{(4)}$, \\ Sr. Fernando Navarro $H^{(5)}$. \\ 1. Medico Radiólogo, Clínica Santa María, Santiago. \\ 2. Becada de Radiología, Universidad de Los Andes, Clínica Santa María, Santiago. \\ 3. Tecnólogo Médico Resonancia Magnética, Clínica Santa María, Santiago. \\ 4. Médico Traumatólogo, Clínica Santa María, Santiago. \\ 5. Administrador PACS, Clínica Santa María, Santiago. \\ e-mail:pyanez@csm.cl
}

TRACTOGRAPHY AND DIFFUSION TENSOR IMAGING IN THE STUDY OF THE MEDIAN NERVE BY MAGNETIC RESONANCE IMAGING. A CASE REPORT

\begin{abstract}
Introduction: Magnetic Resonance Imaging (MRI) is an essential tool for the study of central nervous system disorders. Several studies consider the application of diffusion tensor imaging (DTI) and tractography in the study of peripheral nerves.

Methodology: We performed tractography and DTI in two female patients, one with a confirmed carpal tunnel syndrome and the other in good health conditions. Values of fractional anisotropy (FA) and apparent diffusion coefficient (ADC) were recorded.

Results: The average FA value of the fiber was 0.461 (case) and 0.632 (control). No differences were reported between the average values of the ADC of the fiber.

Discussion: We were able to represent the tract of the median nerve by using a software for tractography reconstruction. The morphology and values of quantitative analysis of DTI depend directly on the reconstruction algorithm used, mainly on the minimum FA value of the fiber to be reconstructed. The value of the FA, obtained by zone, was the most constant variable, being lower for the case.

Keywords: Apparent diffusion coefficient, Diffusion tensor imaging, Fractional anisotropy, Tractography .
\end{abstract}

\section{RESUMEN}

Introducción: La resonancia magnética es una herramienta indispensable en el estudio de enfermedades del sistema nervioso central. Varios estudios consideran la aplicación del tensor de difusión (DTI) y tractografía en el estudio de nervios periféricos. Metodología: Se realizó tractografía y DTI en dos mujeres, una con síndrome del túnel carpiano confirmado y otra sin patología. Se consignaron valores de fracción de anisotropía (FA) y coeficiente de difusión aparente (ADC). Resultados: El valor promedio de FA de la fibra fue 0,461 (caso) y 0,632 (control). No hubo diferencias entre valores promedios de ADC de fibras. Discusión: Se logró representar el tracto del nervio mediano utilizando software de reconstrucción de tractografía. La morfología y valores del análisis cuantitativo del DTI dependen directamente del algoritmo de reconstrucción utilizado, especialmente del umbral mínimo de FA de la fibra a reconstruir. El valor más constante es FA, obtenido por zona, siendo menor en el caso.

Palabras Clave: Coeficiente de difusión aparente, Fracción de anisotropía, Tensor de difusión, Tractografía. 


\section{INTRODUCCIÓN}

La resonancia magnética $(\mathrm{RM})$ es una herramienta indispensable en el estudio de las enfermedades del sistema nervioso central, pero hasta el momento tiene menor aplicación en las enfermedades del sistema nervioso periférico, principalmente debido a su dificultad para delinear adecuadamente los nervios, como consecuencia del bajo contraste que exhiben respecto del tejido que los rodea ${ }^{(1,2)}$. Las primeras neurografías por RM de nervio periférico, realizadas por Filler et al el año 1993, incluían imágenes con T2 FSE $^{(3)}$, más tarde, se agregaron las secuencias T1, T1SE, STIR y T2 FSE con saturación grasa ${ }^{(4,5)}$. El análisis de las imágenes obtenidas es de carácter cualitativo y subjetivo, con resultados poco específicos ${ }^{(1-3)}$, como por ejemplo, una marcada prolongación en T2, que no permite diferenciar edema por denervación, desmielinización o lesión tumoral( ${ }^{(6)}$ lo que ha promovido la búsqueda de nuevos métodos para el estudio de los tractos nerviosos.

Existen en la literatura varios estudios que consideran la aplicación del tensor de difusión (DTI) y de la tractografía en el estudio de nervios periféricos. Dentro de las posibles aplicaciones reportadas se encuentran la evaluación, pronóstico y seguimiento posterior al tratamiento de múltiples patologías, principalmente las neuropatías compresivas de nervios periféricos, como el síndrome del túnel del carpo, lesiones traumáticas y visualización de las fibras en el caso de neoplasias ${ }^{(7-10)}$.

EI DTI es un método no invasivo y cuantitativo, que revela la microestructura y la organización del tejido sobre la base de su capacidad para monitorizar los movimientos aleatorios de las moléculas de agua, propiedad del tejido llamada "difusión", obteniendose un método más objetivo en la medición del daño del nervio ${ }^{(1,2,5)}$.

La difusión existente en las moléculas que forman los nervios periféricos es de tipo preferente, es decir, mayor en la dirección del nervio que en su eje perpendicular, probablemente debido a la capa de mielina que lo envuelve; esta propiedad es conocida como "anisotropía" $(6,9,11)$.

La difusión del tejido es evaluada a través de imágenes potenciadas en difusión (DWI), que nos aportan información de la difusión en una dirección. El índice de medición es la "difusibilidad" que se refiere a la capacidad de una molécula de difundir a través de una membrana.

La RM no es constante cuando se usa para medir la difusión en un medio complejo, como lo es cualquier tejido biológico, y por ello se propone usar el "coeficiente de difusión aparente" (ADC) que es sensible a la composición y a la ultra/microestructura del material donde las moléculas difunden e interactúan entre ellas ${ }^{(12,13)}$.

El tensor de difusión (DTI) es necesario para la caracterización total de la difusión en un tejido; a partir de sus valores se determina el grado y la dirección de preferencia de la difusión de las moléculas que lo componen ${ }^{(9,13)}$. Luego, a través de un software de procesamiento, se reconstruyen con una visión tridimensional los tractos nerviosos, recibiendo el nombre de tractografía.

Existen al menos cuatro índices provenientes del DTI, el principal es la fracción de anisotropía (FA), que tiene un rango de 0 a 1; mientras más cercano a 1 sea el valor numérico de este índice, indica que el tracto en estudio presenta de preferencia movimiento o difusión en una dirección, secundario a indemnidad de la capa de mielina que lo envuelve (valor de FA alto). Mientras más cercano a 0 sea el valor de la FA (valor de FA bajo), reflejará una pérdida de la dirección preferente, probablemente por una pérdida focal o difusa de la capa de mielina ${ }^{(1,2,6,7)}$.

Dentro de las patologías del sistema nervioso periférico, el síndrome del túnel del carpo (STC) es la neuropatía compresiva crónica más frecuente ${ }^{(7,11)}$. Ocurre por un atrapamiento del nervio mediano a nivel de la muñeca, secundario a lo cual se produce un aumento de la presión dentro del túnel, con una obstrucción del retorno venoso, lo que lleva a edema, cambios fibróticos de las capas más externas del nervio, epi y perineuro (Figura 1) y pérdida secundaria de mielina; incluso se ha comprobado degeneración walleriana en cortes histológicos ${ }^{(13)}$.

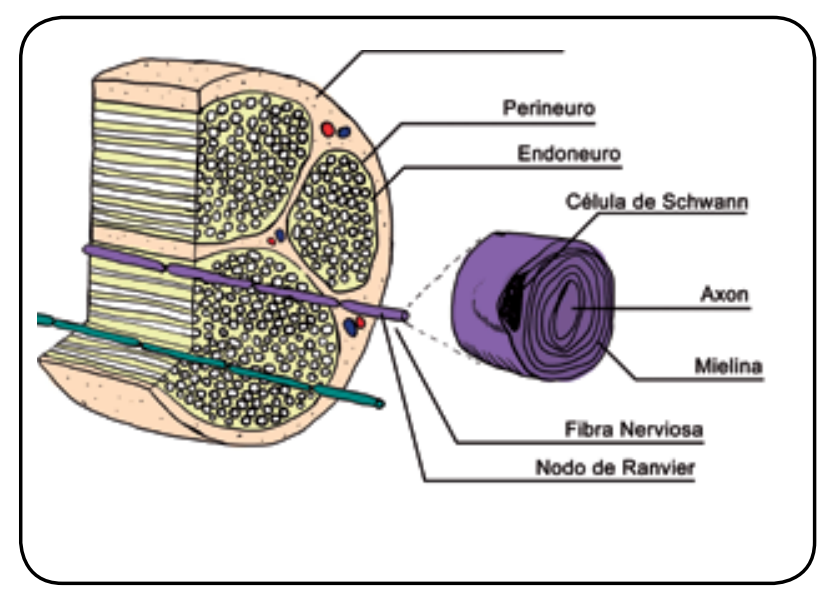

Figura 1. Esquema de la anatomía de un nervio periferico. (adaptado desde referencia 15).

Se describen 5 grados de daño en un nervio; el grado más frecuentemente encontrado en el STC es el primer grado o neuropraxia, en el que existe una pérdida de mielina que se traduce en una menor conducción nerviosa. Los casos más severos pueden llegar a la axonotmesis (grado 2-3) con pérdida de mielina y disrupción, axonal o total, del tracto nervioso (Figura 2). En general, el daño a las 
fibras está relacionado con el grado y duración de la compresión ${ }^{(13-15)}$.

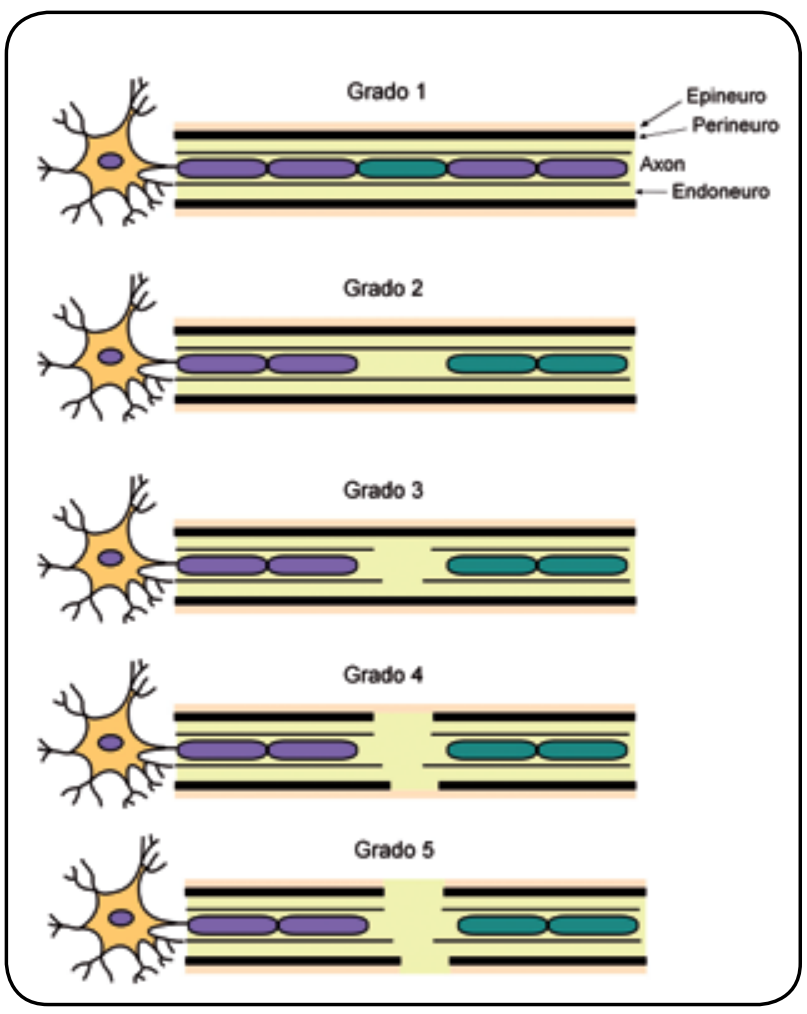

Figura 2. Esquema de nervio con bloqueo de la conducción nerviosa, desde el grado sin cambios morfológicos (neurapraxia, grado 1 de Sunderland) hasta rotura de la fibra nerviosa (grado 5). (adaptado desde referencia 15).

A continuación, a propósito de un caso derivado a nuestro servicio para estudio de neurografía de nervio periférico, se presenta nuestra experiencia en el uso de estas nuevas herramientas diagnósticas.

\section{CASOS}

Se reclutaron dos pacientes de sexo femenino con edad promedio de 30 años, una con diagnóstico clínico y electromiográfico de STC y otra sin patología. En ambas pacientes se descartó uso de medicamentos, cirugías o historia de enfermedades neurológicas. Se denominó caso a la paciente enferma y control a la paciente sana.

El estudio fue realizado en resonador 3 Tesla Philips Achieva X-Series, con gradiente Quasar Dual, $80 \mathrm{mt} / \mathrm{m}$ de amplitud, con slew rate de $100 \mathrm{t} / \mathrm{m} / \mathrm{s}$, utilizando una bobina de muñeca de 8 elementos, factor SENSE de 8, con la paciente en decúbito supino y mano ubicada lateral al cuerpo.

Se realizaron cortes anatómicos T2 de alta resolución con las siguientes características: 60 cortes de 2mm, con TR/TE 6392/93, 2 NEX, FOV DE 100, matriz de 200X 256, TA 4:41 min y secuencia DTI
EPI con TR/TE 4455/56, factor B $1000 \mathrm{~S} / \mathrm{mm} 2,32$ direcciones, NEX 3. TA de 7:34 min.

Luego de adquirida la imagen, se realizó un corregistro automático y se analizó en el software "Extended MR Workspace" Release 2,5.3.1 de Philips Medical Systems.

En el análisis y post proceso de la imagen se realizaron dos pasos esenciales: la reconstrucción del tracto del nervio mediano con el análisis de los valores de la DTI para la fibra (con diferentes algoritmos de reconstrucción predeterminados) y el análisis de tres puntos o áreas focales en el tracto del nervio.

Para realizar el primer paso del post proceso se obtuvo el corte anatómico a nivel del hueso pisiforme en secuencia T2, ubicándose el nervio mediano (Figura 3), luego se correlacionó con el mapa color en los cortes axial y coronal (Figuras 4 y 5), se dibujó en dos puntos distintos una región de interés (ROI) externa al área del nervio, y se trazó el tracto nervioso según el algoritmo de reconstrucción predeterminado (Figuras 6 y 7). Luego se obtuvo los valores numéricos de ADC y FA para la fibra.

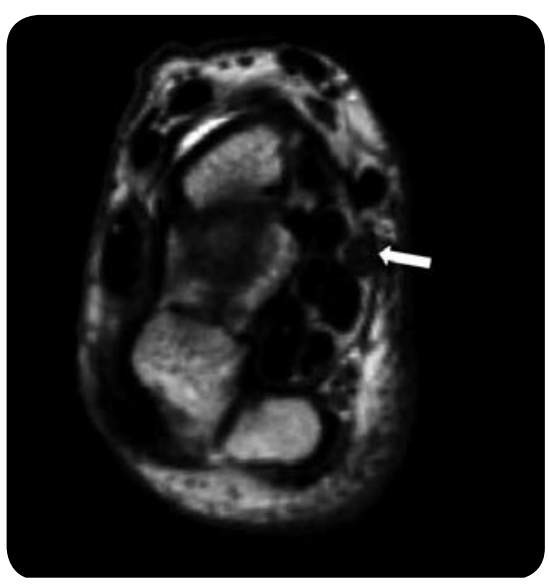

Figura 3. Corte anatómico axial en T2 a nivel a nivel del hueso pisiforme. Se demuestra el nervio mediano (flecha).

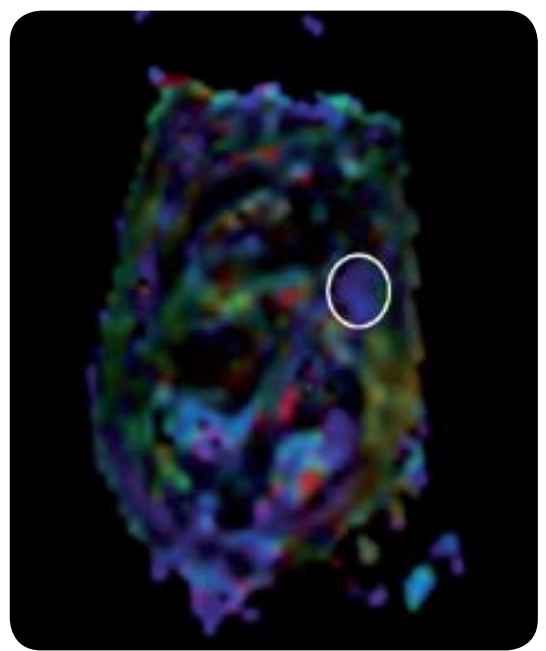

Figura 4. Corte axial al mismo nivel del mapa color para visualizar nervio mediano, previo a reconstrucción. Genera ROI de mayor diámetro del nervio (círculo blanco). 


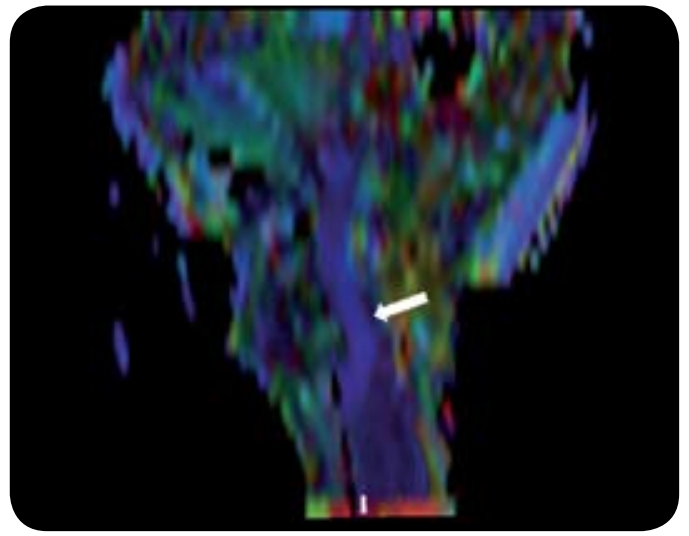

Figura 5. Corte coronal de mapa color para visualización del nervio mediano, previo a generación del tracto (flecha).

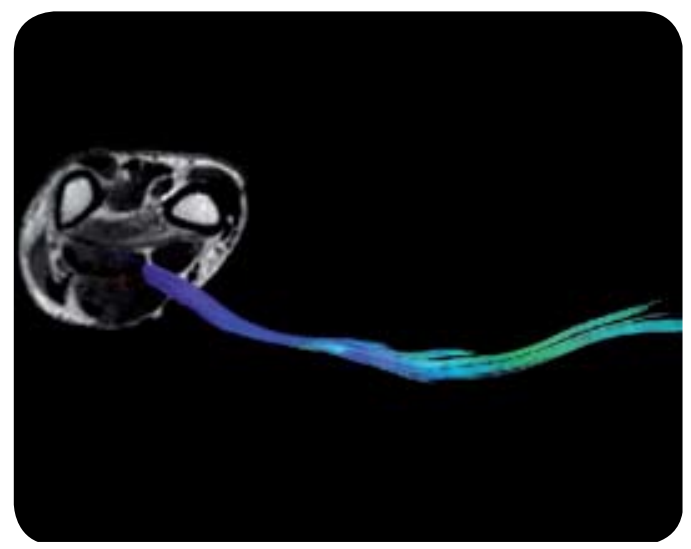

Figura 6. Tractografía del nervio mediano sobre corte anatómico axial T2, en una reconstrucción tridimensional.

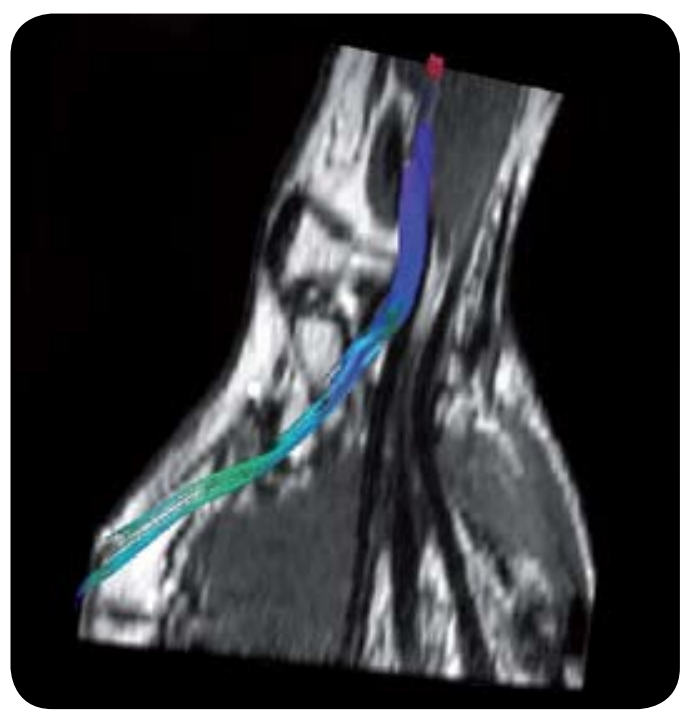

Figura 7. Tractografía de nervio mediano sobre un corte anatómico coronal T2.
Para el segundo paso, se ubicó el nervio en cortes anatómicos T2, sobre el cual se ubicó en el mapa color axial y coronal, realizándose mediciones en tres puntos focales del trayecto del nervio, realizando ROls con diámetros menores al área del nervio. Un ROI en el antebrazo, aproximadamente a $5 \mathrm{~cm}$ proximal al túnel del carpo (zona 1), en la región del túnel a la altura del hueso pisiforme (zona 2) y un tercer ROI a 1,5 cm caudal al túnel (zona 3) (Figuras 8 y 9), obteniendo valores de ADC y FA para cada una de las zonas estudiadas.

Los resultados obtenidos en las mediciones del caso y del control son mostrados en las tablas 1 y 2 . El nervio mediano pudo ser identificado como una formación redondeada o de forma oval, de color azul, visible tanto en el corte axial como en la reconstrucción coronal del mapa color. En ambos casos hubo una correlación en la ubicación del nervio con los cortes anatómicos en T2.

En ambos sujetos de estudio se realizó la reconstrucción de la fibra nerviosa con tractografía, que se correlaciona con la ubicación de ella en cortes anatómicos y en el mapa color.

La fibra reconstruida no presenta diferencias en la evaluación visual, con respecto a la morfología del tracto entre el caso y el control.

El valor promedio de la FA de la fibra del caso fue 0,461 , notoriamente menor al caso control que alcanzó a 0,632. No se visualizaron diferencias entre los valores promedios del ADC de la fibra.

Con respecto al análisis entre las tres zonas estudiadas, hay diferencias principalmente en la FA medida en la zona del túnel del carpo en caso y control, con FA de 0,426 versus 0,761 . El valor de $F A$ de ambas pacientes entre las zonas $1 \mathrm{y}$, de las zonas 3 entre sí, no presentan diferencias significativas. En el caso hay una reducción de alrededor de un $40 \%$ de la FA entre la zona 1 y 2 . En el control, la FA en la zona 2 se encuentra levemente aumentada con respecto a la zona $1(0,761$ versus 0,739$)$.

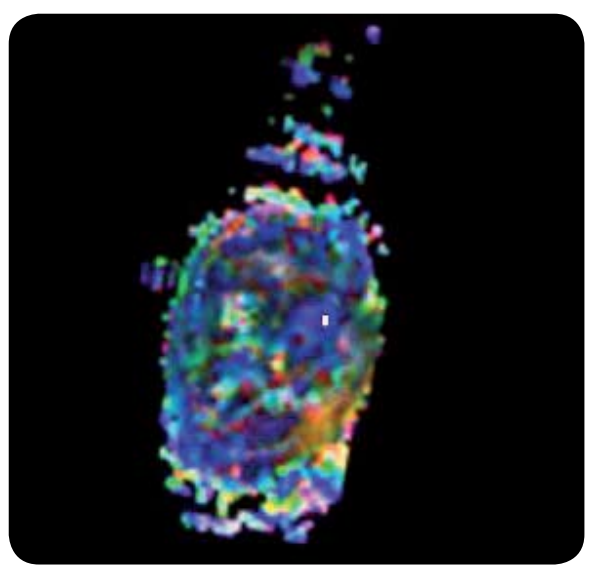

Figura 8. Corte axial mapa color de FA a nivel focal (zona 2) con ROI de menos diámetro que área del nervio. (Círculo pequeño blanco). 
Tabla I. Promedios de ADC y de valores máximos de FA en nervio mediano, antebrazo y muñeca $(\bar{X} \pm D S)$.

\begin{tabular}{|l|lr|lc|lc|}
\multicolumn{2}{|l}{ Zona 1 (antebrazo) } & \multicolumn{2}{l}{ Zona 2 (túnel carpiano) } & \multicolumn{2}{l}{ Zona 3 (muñeca distal) } \\
\hline & FA & ADC & FA & ADC & FA & ADC \\
\hline Caso & $0.688 \pm 0$ & $1.007 \pm 0$ & $0.426 \pm 0.076$ & $1.034 \pm 0.038$ & $0.585 \pm 0.034$ & $0.948 \pm 0.120$ \\
Control & $0.739 \pm 0$ & $1.042 \pm 0$ & $0.761 \pm 0$ & $0.649 \pm 0$ & $0.619 \pm 0$ & $0.638 \pm 0$ \\
& & & & & & \\
\hline
\end{tabular}

FA: Fracción de Anisotropía ; ADC: Coeficiente de difusión aparente $\left(\times 10^{-3} \mathrm{~mm}^{2} / \mathrm{s}\right) ; \bar{x}$ : Promedio; DS: Desviación estándar; Caso: Paciente enfermo; Control: Sano.

Tabla II. Promedio del Factor de Anisotropía, promedio de coeficiente de difusión aparente y longitud de fibra del nervio mediano.

\begin{tabular}{|l|c|c|c|}
\multicolumn{2}{c}{ FA } & ADC $\left(\times 10^{-3} \mathrm{~mm}^{2} / \mathrm{s}\right)$ & Longitud $(\mathrm{mm})$ \\
\hline Fibra Caso & $0.632 \pm 0.198$ & 0.984 & 56 \\
Fibra Control & $0.461 \pm 0.117$ & $1.142 \pm 0.357$ & $80.96 \pm 29.34$ \\
\hline
\end{tabular}

FA: Fracción de Anisotropía; ADC: Coeficiente de difusión aparente $\left(\times 10^{-3} \mathrm{~mm}^{2} / \mathrm{s}\right)$; Caso: Paciente enfermo; Control: Sano

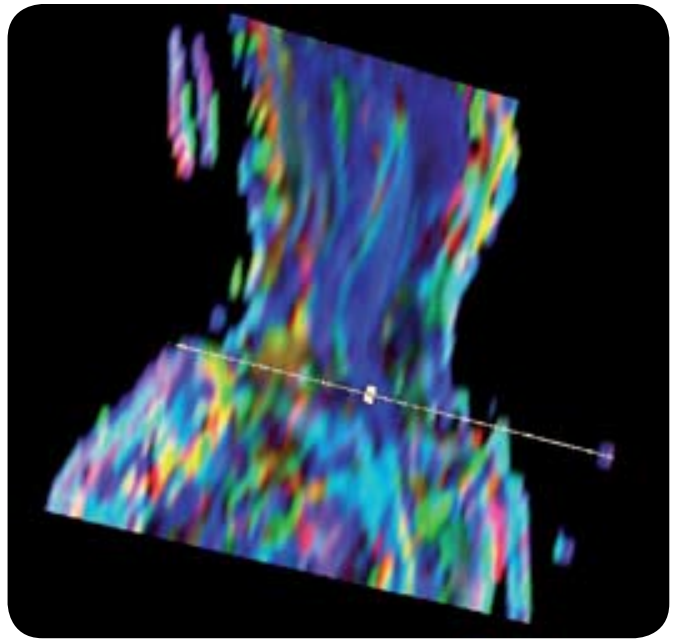

Figura 9. Corte coronal mapa color, visualizándose el nervio mediano como guía, medición de la FA a nivel focal.

\section{DISCUSIÓN}

El estudio de nervio periférico por RM (neurografía de nervio periférico) se obtenía en sus inicios con secuencias potenciadas en T2 Fat sat (1993) ${ }^{(3)}$ y STIR $(2002)^{(5)}$; éstas secuencias anatómicas tienen la desventaja de una pobre delineación del nervio, debida a la baja resolución de contraste con el tejido adyacente y la poca especificidad de los hallazgos en T2, observando edema en patología compresiva, regeneración y tumores ${ }^{(1,6)}$.

En los últimos años se han publicado varios trabajos acerca del estudio de nervios periféricos a través del tensor de difusión y tractografía. En un estudio experimental realizado por Takagi et al, $2009^{(1)}$, con un resonador de 7 Tesla, se demostró que existe una correlación entre los cambios histológicos después de una lesión, como por ejemplo daño axonal, desmielinización, degeneración walleriana, y el factor de anisotropía, que se encuentra afectado en el epicentro de la lesión ${ }^{(1-3)}$.

Nuestro grupo, al igual que los estudios mencionados previamente, pudo representar el tracto nervioso del nervio mediano al utilizar un software de reconstrucción de tractografía. La morfología y valores del análisis cuantitativo del DTI dependen directamente del algoritmo de reconstrucción utilizado, en especial del valor umbral mínimo de la FA de la fibra a reconstruir, que determina el valor promedio de la FA del tracto nervioso reconstruido (Figuras 10 y 11).

El valor de la FA de la fibra es menor en el caso, paciente con STC documentado, lo que reflejaría la presencia de desmielinización y daño axonal. No se encontraron diferencias en los valores de ADC, lo que refleja que no existe un trastorno en la difusibilidad, hallazgo que se observaría en presencia de un daño del epineuro, en los grados más severos.

Los valores encontrados en el análisis de la FA por zonas, demostraron una reducción del FA del nervio mediano en la zona del túnel del carpo, mayor a un $40 \%$, lo que refleja disminución de la anisotropía preferente en la zona de compresión, probablemente secundaria a pérdida de mielina o a daño axonal(10). 


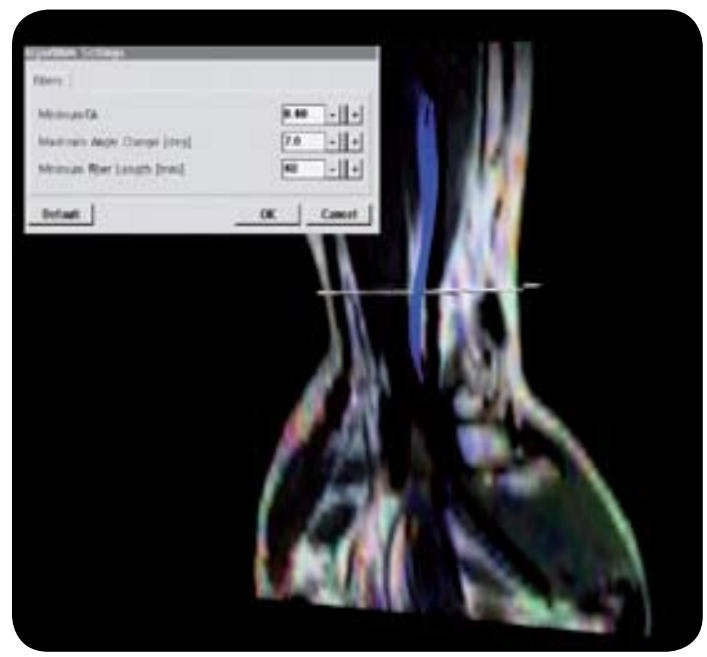

Figura 10. Algoritmo de reconstrucción con FA de 0,4.

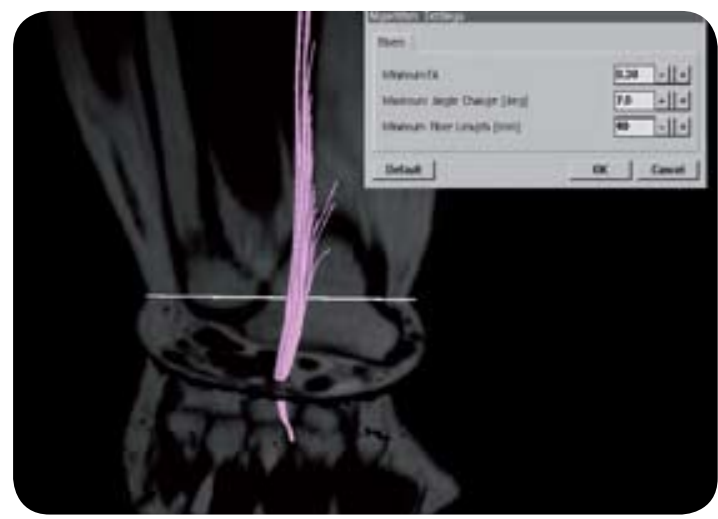

Figura 11. Algoritmo de reconstrucción con FA de 0,3. Nótese el cambio en la morfología de la fibra, en comparación con la figura 10.

\section{BIBLIOGRAFÍA}

1. Takagi T, Nakamura M, Yamada M, Hikishima K, Momoshima S, Fujiyoshi K, et al. Visualization of peripheral nerve degeneration and regeneration: monitoring with diffusion tensor tractography. Neuroimage. 2009; 44(3): 884-92.

2. Meek MF, Stenekes MW, Hoogduin HM, Nicolai JP. In vivo three-dimensional reconstruction of human median nerves by diffusion tensor imaging. Exp Neurol. 2006;
198(2):479-82.

3. Filler AG, Howe FA, Hayes CE, Kliot M, Winn HR, Bell $\mathrm{BA}$, et al. Magnetic resonance neurography. Lancet 1993; 341: 659-661.

4. Filler AG, Maravilla KR, Tsuruda JS. MR neurography and muscle MR imaging for image diagnosis of disorders affecting the peripheral nerves and musculature. Neurol. Clin. 2004; 22: 643-682 vi-vii.

5. Grant GA, Britz GW, Goodkin R, Jarvik JG, Maravilla $\mathrm{K}$, Kliot $\mathrm{M}$. The utility of magnetic resonance imaging in evaluating peripheral. nerve disorders. Muscle Nerve 2002; 25: 314-331.

6. Hiltunen J, Suortti T, Arvela S, Seppä M, Joensuu R, Hari R. Diffusion tensor imaging and tractography of distal peripheral nerves at 3 T. Clin Neurophysiol. 2005; 116(10): 2315-23.

7. Merlini L, Viallon M, De Coulon G, Lobrinus JA, Vargas MI. Pediatr Radiol. 2008; 38(9): 1009-12. MRI neurography and diffusion tensor imaging of a sciatic perineuroma in a child.

8. Andreisek G, White LM, Kassner A, Tomlinson G, Sussman MS. Diffusion tensor imaging and fiber tractography of the median nerve at $1.5 \mathrm{~T}$ : optimization of b value. Skeletal Radiol. 2009; 38(1): 51-9.

9. Khalil C, Hancart C, Le Thuc V, Chantelot C, Chechin $D$, Cotten A. Diffusion tensor imaging and tractography of the median nerve in carpal tunnel syndrome: preliminary results. Eur Radiol. 2008; 18(10): 2283-91.

10. Stein D, Neufeld A, Pasternak O, Graif M, Patish H, Schwimmer E, Ziv E, Assaf Y. Diffusion tensor imaging of the median nerve in healthy and carpal tunnel syndrome subjects. J Magn Reson Imaging. 2009; 29(3): 657-62.

11. Kabakci N, Gürses B, Firat Z, Bayram A, Ulu AM, Kovanlikaya $A$, et al. Diffusion tensor imaging and tractography of median nerve: normative diffusion values. AJR Am J Roentgenol. 2007; 189(4): 923-7.

12. Skorpil M, Karlsson M, Nordell A. Peripheral nerve diffusion tensor imaging. Magn Reson Imaging. 2004; 22(5): 743-5.

13. Basser, PJ, Mattiello J, LeBihan D. MR diffusion tensor spectroscopy and imaging. Biophys. J. 1994; 66: 259267.

14. Kabakci NT, Kovanlikaya A, Kovanlikaya I. Tractography of the median nerve. Semin Musculoskelet Radiol. 2009; 13(1): 18-23.

15. Moller A. Anatomy and Physiology of Peripheral Nerves. Moller A. Intraoperative Neurophysiological Monitoring. 2nd Edición. Totowa, NJ: Humana Press. 2006; 221227. 